\title{
Bayes Estimation under a Finite Mixture of Truncated Generalized Cauchy Distributions Based On Censored Data with Application
}

\author{
Saieed F Ateya ${ }^{1,2 *}$ and Hesah A Al Khald ${ }^{1}$ \\ ${ }^{1}$ Department of Mathematics \& Statistics, Taif University, Saudi Arabia \\ ${ }^{2}$ Department of Mathematics, Assiut University, Egypt \\ Submission: December 04, 2017; Published: February 19, 2018 \\ *Corresponding author: Saieed F Ateya, Department of Mathematics \& Statistics, Taif University, Saudi Arabia, Tel: 9.66545E+11; \\ Email: 4270176@gmail.com
}

Abstract

In this paper, the Bayes estimates (BE's) of the parameters, reliability and hazard rate functions of a finite mixture of truncated generalized Cauchy distributions are obtained based on type-I, type-II and progressively type-II censored samples. A simulation study is carried out to study the behaviour of the mean squared errors (MSE's) of the estimates. All previous parameters and functions are obtained based on generated type-I, type-II and progressively type-II censored samples generated from a real data set as illustrative application.

Keywords: Truncated generalized Cauchy distribution; Bayes estimation; MCMC algorithm; Finite mixture models; Type-I censoring; Type-Il censoring; Progressively type-II censoring

Abbreviations: BE's: Bayes Estimates; MSE's: Mean Squared Errors; TGCD: Truncated Generalized Cauchy Distribution; PDF: Probability Density; CDF: Cumulative Distribution Function; SF: Survival Function; HRF: Hazard Rate Function; LF: Likelihood Function

\section{Introduction}

The Cauchy distribution is a symmetric distribution with bell shaped density function as the normal distribution but with a greater probability mass in the tails. The distribution is often used in the cases which arise in outlier analysis. The Cauchy distribution has received applications in many areas, including biological analysis, clinical trials, stochastic modelling of decreasing failure rate life components, queuing theory, and reliability. For data from these areas, there is no reason to believe that empirical moments of any order should be infinite. Thus, the choice of the Cauchy distribution as a model is unrealistic since its moments of all orders are not infinite. The introduced truncated generalized Cauchy distribution (TGCD) can be a more appropriate model for the kind of data mentioned. For more details about Cauchy and truncated generalized Cauchy distributions, see the book by Johnson et al. [1] which covers the Cauchy distribution in many of its aspects starting from the history, properties, developments and applications up to the most recent research done in the subject matter, to the date of the book's publication. Also see, Ateya \& AL-Hussaini [2] and Ahsanullah [3] which studied the TGCD extensively. The probability density (PDF), cumulative distribution function (CDF), survival function (SF) and hazard rate function (HRF) of the TGCD with parameters $(\alpha, \beta, \gamma)$ are given, respectively, by

$$
\begin{aligned}
& f(t ; \alpha, \beta, \gamma)=\frac{2}{\sqrt{\pi}} \frac{\Gamma\left(\alpha+\frac{1}{2}\right)}{r \Gamma(\alpha)}\left[1+\left(\frac{t-\beta}{r}\right)^{2}\right]^{-\alpha-\frac{1}{2}}, t>\beta(\beta, \gamma, \alpha>0) . \\
& \mathrm{F}(\mathrm{t} ; \alpha, \beta, \gamma)=\frac{2}{\sqrt{\delta}} \frac{\tilde{\mathrm{A}}\left(\alpha+\frac{1}{2}\right)}{r \tilde{\mathrm{A}}(\alpha)} \int_{0}^{\tan ^{-1}(\mathrm{t}-\beta) / r}(\cos \varphi)^{2 \alpha-1} \mathrm{~d} \varphi \\
& \mathrm{S}(\mathrm{t} ; \alpha, \beta, \gamma)=1-\left(\left(\frac{2}{\sqrt{\pi}} \frac{\Gamma\left(\alpha+\frac{1}{2}\right)}{\mathrm{r} \Gamma(\alpha)} \int^{\left.\tan ^{-\left(\frac{t(-\beta)}{r}\right)}(\cos \varphi)^{2 \alpha-1} \mathrm{~d} \varphi\right)}\right.\right.
\end{aligned}
$$

And

$$
\mathrm{h}(\mathrm{t} ; \alpha, \beta, \gamma)=\frac{\left(\frac{2}{\sqrt{\pi}} \frac{\left(\alpha+\frac{1}{2}\right)}{r \Gamma(\alpha)}\left[1+\left(\frac{t-\beta}{r}\right)^{2}\right]^{\alpha-\frac{1}{2}}\right)}{\left(1-\frac{2}{\sqrt{\pi}} \frac{\left(\alpha+\frac{1}{2}\right)}{r \Gamma(\alpha)} \int^{\left.\tan ^{-\left(\frac{1-\beta}{r}\right)}(\cos \varphi)^{2 \alpha-1} \mathrm{~d} \varphi\right)}\right.}
$$

The mixture models are very important in the theoretical and applied fields especially in case of the heterogeneous 
population. For details about mixture models, see McLachlan \& Peel [4], Titterington, Smith \& Makov [5], Bozidar et al. [6] and Satheesh \& Manju [7]. A random variable T is said to have a finite mixture of TGCD's with parameters $\theta_{j}=\left(\alpha_{j}, \beta_{j}, \gamma_{j}\right), j=1,2, \ldots, k$, if its PDF is given by

$$
\mathrm{f}_{\Theta}(\mathrm{t})=\sum_{\mathrm{j}=1}^{k} \mathrm{p}_{\mathrm{j}} \mathrm{f}_{\mathrm{j}}\left(\mathrm{t} ; \theta_{\mathrm{j}}\right)
$$

Where, $\Theta=\left(\theta_{1}, \theta_{2}, \ldots, \theta_{\mathbf{k}}, \mathrm{p}_{1}, \ldots, \mathrm{p}_{\mathrm{k}}\right), \mathrm{p}_{\mathrm{j}} \geq 0$ and $\sum_{J=1}^{k} \mathrm{p}_{\mathrm{j}}=1$.

The corresponding CDF, SF and HRF are given by

$$
\begin{array}{r}
\mathrm{F}_{\Theta}(t)=\sum_{j=1}^{k} \mathrm{p}_{\mathrm{j}} \mathrm{F}_{\mathrm{j}}\left(\mathrm{t} ; \theta_{\mathrm{j}}\right), \\
\mathrm{S}_{\Theta}(\mathrm{t})=\sum_{j=1}^{k} \mathrm{p}_{\mathrm{j}} \mathrm{S}_{\mathrm{j}}\left(\mathrm{t} ; \theta_{\mathrm{j}}\right),
\end{array}
$$

And,

$$
h_{\Theta}(t)=\frac{f_{\Theta}(t)}{S_{\Theta}(t)}
$$

Where, $\mathrm{f}_{\mathrm{j}}\left(\mathrm{t} ; \theta_{\mathrm{j}}\right), \mathrm{F}_{\mathrm{j}}\left(\mathrm{t} ; \theta_{\mathrm{j}}\right)$ and $\mathrm{S}_{\mathrm{j}}\left(\mathrm{t} ; \theta_{\mathrm{j}}\right)$ can be obtained from equations (1.1)-(1.3) after replacing $\theta=(\alpha, \beta, \gamma)$ by $\theta_{\mathrm{j}}=\left(\alpha_{\mathrm{j}}, \beta_{\mathrm{j}}, \gamma_{\mathrm{j}}\right)$.

\section{Prior analysis and some important algorithms}

In this section, a suggested prior and some important algorithms will be introduced.

Prior analysis: Suppose that the prior belief of the experimenter is measured by a prior PDF $\pi\left(p, \alpha_{1}, \alpha_{2}, \gamma_{1}, \gamma_{2}, \beta\right)$ constructed as follows:

$$
\begin{aligned}
& \pi\left(p, \alpha_{1}, \alpha_{2}, \gamma_{1}, \gamma_{2}, \beta\right)=\pi_{1}(p) \pi_{2}\left(\alpha_{1}, \gamma_{1}\right) \pi_{3}\left(\alpha_{2}, \gamma_{2}\right) \pi_{4}(\beta)= \\
& \pi_{1}(p) \pi_{21}\left(\gamma_{1} \mid \alpha_{1}\right) \pi_{22}\left(\alpha_{1}\right) \pi_{31}\left(\gamma_{2} \mid \alpha_{2}\right) \pi_{32}\left(\alpha_{2}\right) \pi_{4}(\beta)
\end{aligned}
$$

Suppose that $\pi_{1}(p)$ is Beta $\left(c_{1}, c_{2}\right), \pi_{21}\left(\gamma_{1} \mid \alpha_{1}\right)$ is Gamma $\left(c_{3}, \alpha_{1}\right), \pi_{22}\left(\alpha_{1}\right)$ is Gamma $\left(c_{4}, c_{5}\right), \pi_{31}\left(\gamma_{2} \mid \alpha_{2}\right)$, is Gamma $\left(c_{4}, c_{5}\right), \pi_{32}\left(\gamma_{2} \mid \alpha_{2}\right)$ is Gamma $\left(c_{7}, c_{8}\right)$ and finally $\pi_{4}(\beta)$ is Gamma

$\left(c_{9}, c_{10}\right)$ with respective densities

$$
\begin{aligned}
& \pi_{1}(p) \propto p^{c_{1}-1}(1-p)^{c_{2}-1}, 0 \leq p \leq 1,\left(c_{1}, c_{2}>0\right) \\
& \pi_{21}\left(\gamma_{1} \mid \alpha_{1}\right) \propto \alpha_{1}^{c_{3}} \gamma_{1}^{c_{3}-1} e^{-\gamma_{1} \alpha_{1}}, \alpha_{1}, \gamma_{1}>0,\left(c_{3}>0\right) \\
& \pi_{22}\left(\alpha_{1}\right) \propto \alpha_{1}^{c_{4}-1} e^{-c_{5} \alpha_{1}}, \alpha_{1}>0,\left(c_{4}, c_{5}>0\right), \\
& \pi_{31}\left(\gamma_{2} \mid \alpha_{2}\right) \propto \alpha_{2}^{c_{6}} \gamma_{2}^{c_{6}-1} e^{-\gamma_{2} \alpha_{2}}, \alpha_{2}, \gamma_{2}>0,\left(c_{6}>0\right) \\
& \pi_{32}\left(\alpha_{2}\right) \propto \alpha_{2}^{c_{7}-1} e^{-c_{8} \alpha_{2}}, \alpha_{2}>0,\left(c_{7}, c_{8}>0\right) \\
& \pi_{4}(\beta) \propto \beta^{c_{9}-1} e^{-c_{10} \beta} \cdot \beta>,\left(c_{7}, c_{8}>0\right)
\end{aligned}
$$

From equations (2.2) to (7.7) in equation (2.1), we can write the prior PDF of the parameters $\left(p, \alpha_{1}, \alpha_{2}, \gamma_{1}, \gamma_{2}, \beta\right)$ as follows:

$$
\begin{aligned}
& \pi\left(\rho, \alpha_{1}, \alpha_{2}, \gamma_{1}, \gamma_{2}, \beta\right) \alpha \rho^{c_{1}-1}(1-p)^{c_{2}-1} \alpha_{1}^{c_{3}+c_{4}-1} \alpha_{2}^{c_{6}+c_{7}-1} \gamma_{1}^{c_{3}-1} \gamma_{2}^{c_{6}-1} \beta^{c_{9}-1} \\
& \exp \left\{\left[\alpha_{1}\left(\gamma_{1}+\mathrm{c}_{5}\right)+\alpha_{2}\left(\gamma_{2}+\mathrm{c}_{8}\right)+c_{10} \beta\right]\right\} \\
& 0 \leq \mathrm{p} \leq 1, \alpha_{1}, \alpha_{2}, \gamma_{1}, \gamma_{2}, \beta>0,\left(c_{1}, c_{2}, c_{3}, c_{4}, c_{5}, c_{6}, c_{7}, c_{8}, c_{9}, c_{10}>0\right)
\end{aligned}
$$

Where, $c_{1}, c_{2}, c_{3}, c_{4}, c_{5}, c_{6}, c_{7}, c_{8}, c_{9}$ and are the prior parameters.

\section{Gibbs sampler}

Gibbs Sampler is a method used to generate a random sample $\theta^{1}, \theta^{2}, \ldots, \theta^{m}$ from the posterior PDF $\pi^{*}(\theta \mid t)$ as follows:

Let $\left.\theta^{\circ}=\mathrm{e}_{1}^{\circ}, \ldots, \mathrm{e}_{k}\right)$ be an initial values [may be actual values of parameters, or may be the estimated values using any method]

2- Generate $\theta_{1}^{1}$ from $\pi^{*}\left(\theta_{1} \mid \theta_{2}^{\circ}, \theta_{3}^{\circ}, \ldots, \theta_{k}^{\circ}, t\right)$.

3- Generate $\theta_{1}^{1}$ from $\pi^{*}\left(\theta_{2} \mid \theta_{1}^{1}, \theta_{3}^{\circ}, \ldots, \theta_{k}^{\circ}, t\right)$.

4- Generate $\theta_{1}^{1}$ from $\pi^{*}\left(\theta_{i} \mid \theta_{1}^{1}, \theta_{2}^{1}, \ldots, \theta_{i-1}^{1}, \theta_{i+1}^{\circ}, \ldots, \theta_{k}^{\circ}, t\right)$

5- Generate $\theta_{k}^{1}$ from $\pi^{*}\left(\theta_{k} \mid \theta_{1}^{1}, \theta_{2}^{1}, \ldots, \theta_{k-1}^{1}, t\right)$ so we generate $\theta^{1}=\left(\theta_{1}^{1}, \ldots, \theta_{k}^{1}\right)$

6- Repeat steps 1-5 m times we get $\theta^{1}, \theta^{2}, \ldots, \theta^{\mathbf{m}}$.

\section{Metropolis-Hastings algorithm}

Is a method used to generate a number $\theta_{i}^{j}$ from the posterior PDF $\pi^{*}\left(\theta_{i} \mid \theta_{1}^{j}, \theta_{2}^{j}, \ldots, \theta_{i-1}^{j}, \theta_{i+1}^{j-1}, \ldots, \theta_{k}^{j-1}, x\right)$. This method can be summarized in the following steps:

1- Generate $\theta_{i}^{*}$ from a suitable PDF $f(\theta)$

2- $A^{*}=\min \{1, A\}$,

$$
A=\frac{\pi^{*}\left(\theta_{1}^{j}, \theta_{2}^{j}, \ldots, \theta_{i-1}^{j}, \theta_{i}^{*}, \theta_{i+1}^{j-1}, \ldots, \theta_{k}^{j-1}, t\right) f\left(\theta_{i}^{*}\right)}{\pi^{*}\left(\theta_{1}^{j}, \theta_{2}^{j}, \ldots, \theta_{i-1}^{j}, \theta_{i}^{j-1}, \ldots, \theta_{k}^{j-1}, t\right) f\left(\theta_{i}^{j-1}\right)}
$$

3- Generateu from $u(0,1)$

4- If $A<U$, then accept $\theta_{i}^{*}$ as $\theta_{i}^{j}$, else $\theta_{i}^{*} \rightarrow \theta_{i}^{j-1}$ go to step1.

\section{Markov chain monte carlo (MCMC) method}

Let $\theta=\left(\theta_{1}, \theta_{2}, \ldots, \theta_{k}\right)$ be a parameters vector with a posterior PDF $\pi^{*}(\theta \mid t), \quad t=\left(t_{1}, t_{2}, \ldots, t_{n}\right)$ the vector of observations. If $\theta^{1}, \theta^{2}, \ldots, \theta^{m}$, where $\theta^{i}=\left(\theta_{1}^{i}, \theta_{2}^{i}, \ldots, \theta_{k}^{i}\right)$ is a random sample of size $\mathrm{m}$ generated from $\pi^{*}(\theta \mid t)$, then the BE of a function $u\left(\theta^{i}\right)$ based on squared error (SE) loss functions is given by

$$
\widehat{u}_{B S}(\theta)=\frac{1}{m} \sum_{i=1}^{m} u\left(\theta^{i}\right),
$$

To generate from the posterior PDF $\pi^{*}(\theta \mid t)$, we will use Gibbs sampler and Metropolis-Hastings techniques. For more details about the MCMC method, see, for example Jaheen \& Al-Harbi [8], Press[9], Upadhyaya et al. [10] and Upadhyaya \& Gupta [11]. 


\section{Bayes estimation}

In this section, the BE's of all parameters, survival and hazard rate functions will be estimated based on type-I, type-II and progressive type-II censoring schemes.

\section{Bayes Estimation Based on type-I censoring scheme}

Suppose that we have $\mathrm{n}$ items from a finite mixture TGCD's, with equal location parameters $\left(\beta_{1}=\beta_{2}=\beta\right)$. All items are put on a life testing experiment. Suppose that $\mathrm{r}$ units have failed during the interval $\left(0, t_{0}\right)$ and $(n-r)$ units are still active, where $t_{0}$ is a predetermined time. Let $t_{1}, \ldots, t_{n}$ be a random sample from the mixed population. The exact lifetime of an item will be observed only if $t_{i} \leq t_{0}, i=1,2, \ldots, n$. This is known as type-I censored sample. The likelihood function(LF) based on type-I censored sample, see Lawless[12], may be written as

$$
\mathrm{L}(\theta \mid t)=\prod_{\mathrm{i}=1}^{n}\left[\mathrm{f}_{\Theta}\left(\mathrm{t}_{\mathrm{i}}\right)\right]^{\ddot{a}_{\mathrm{i}}}\left[\mathrm{S}_{\Theta}\left(\mathrm{t}_{0}\right)\right]^{1-\ddot{\mathrm{a}}_{\mathrm{i}}}
$$

Where, $\mathrm{f}_{\grave{\mathbf{E}}}\left(\mathrm{t}_{\mathrm{i}}\right)$ and $\mathrm{S}_{\dot{\mathbf{E}}}\left(\mathrm{t}_{\mathrm{o}}\right)$ are defined in (1.5) and (1.7) after replacing t by $t_{i}$ and $t_{0}$ respectively,

$$
t=\left(\mathrm{t}_{1}, \mathrm{t}_{2}, \ldots ., \mathrm{t}_{\mathrm{n}}\right)
$$

And $\ddot{a}_{\mathrm{i}}$ is an indicator function, given by

$$
\ddot{a}_{\mathrm{i}}= \begin{cases}1, & \mathrm{t}_{\mathrm{i}} \leq \mathrm{t}_{0}, \\ 0, & \mathrm{t}_{\mathrm{i}}>\mathrm{t}_{0} .\end{cases}
$$

Using the LF (3.1) and the prior (2.8), the posterior PDF of the parameters $\left(p, \alpha_{1}, \alpha_{2}, \gamma_{1}, \gamma_{2}, \beta\right)$ can be written as

$$
\begin{aligned}
& \pi^{*}(\theta \mid t) p^{c_{1}-1}(1-p)^{c_{2}-1} \alpha_{1}^{c_{3}+c_{4}-1} \alpha_{2}^{c_{6}+c_{c}-1} \gamma_{1}^{c_{3}-1} \gamma_{2}^{c_{6}-1} \beta^{c_{5}-1} \\
& \exp \left\{\left[\alpha_{1}\left(\gamma_{1}+c_{5}\right)+\alpha_{2}\left(\gamma_{2}+c_{8}\right)+c_{10} \beta\right]\right\} \Pi_{i=1}^{n}\left[f\left(t_{i} ; \theta\right)\right]^{\delta_{i}}\left[S\left(t_{0} ; \theta\right)\right]^{1-\delta_{i}}
\end{aligned}
$$

To estimate the parameters and functions $p, \alpha_{1}, \alpha_{2}, \gamma_{1}, \gamma_{2}, \beta$, survival and hazard rate functions at time $t^{*}$, we define a function $u\left(p, \alpha_{1}, \alpha_{2}, \gamma_{1}, \gamma_{2}, \beta\right)$ as

$u\left(p, \alpha_{1}, \alpha_{2}, \gamma_{1}, \gamma_{2}, \beta\right)=p^{\delta_{1}} \alpha_{1}^{\delta_{2}} \alpha_{2}^{\delta_{3}} \gamma_{1}^{\delta_{4}} \gamma_{2}^{\delta_{5}} \beta^{\delta_{6}}\left(S\left(t^{*}\right)\right)^{\delta_{7}}\left(\mathrm{~h}\left(t^{*}\right)\right)^{\delta_{8}}$.

The BE of $u\left(p, \alpha_{1}, \alpha_{2}, \gamma_{1}, \gamma_{2}, \beta\right)$ is obtained in five cases:

When $\delta_{1}=1, \delta_{2}=0=\delta_{3}=\delta_{4}=\delta_{5}=\delta_{6}=\delta_{7}=\delta_{8}$, which is equivalent to estimating $p$.

When $\delta_{2}=1, \delta_{1}=0=\delta_{3}=\delta_{4}=\delta_{5}=\delta_{6}=\delta_{7}=\delta_{8}$, which is equivalent to estimating $\alpha_{1}$.

When $\delta_{3}=1, \delta_{1}=0=\delta_{2}=\delta_{4}=\delta_{5}=\delta_{6}=\delta_{7}=\delta_{8}$, which is equivalent to estimating $\alpha_{2}$

When $\delta_{4}=1, \delta_{1}=0=\delta_{2}=\delta_{3}=\delta_{5}=\delta_{6}=\delta_{7}=\delta_{8}, \quad$ which is equivalent to estimating $\gamma_{1}$.

When $\delta_{5}=1, \delta_{1}=0=\delta_{2}=\delta_{3}=\delta_{4}=\delta_{6}=\delta_{7}=\delta_{8}$, which is equivalent to estimating $\gamma_{2}$

When $\delta_{6}=1, \delta_{1}=0=\delta_{2}=\delta_{3}=\delta_{4}=\delta_{5}=\delta_{7}=\delta_{8}, \quad$ which is equivalent to estimating $\beta$.
When $\delta_{7}=1, \delta_{1}=0=\delta_{2}=\delta_{3}=\delta_{4}=\delta_{5}=\delta_{6}=\delta_{8}, \quad$ which is equivalent to estimating $S\left(t^{*}\right)$.

When $\delta_{8}=1, \delta_{1}=0=\delta_{2}=\delta_{3}=\delta_{4}=\delta_{5}=\delta_{6}=\delta_{7}, \quad$ which is equivalent to estimating $h\left(t^{*}\right)$.

Then, MCMC algorithm will be used to estimate all mentioned parameters and functions

\section{Bayes estimation based on type-II censoring scheme}

Assume that we put items from a finite mixture of TGCD's in a life testing experiment. Instead of continuing until all $n$ items have failed, the experiment is terminated at the time of the $\mathrm{r}^{\text {th }}$ item failure. Such test can save time and money, since it could take a very long time for all items to fail. Suppose that $t_{1}<t_{2}<\ldots<t_{r}$ is a censored data of size $r$ obtained from a life test on $n$ items (type-II censored data) whose life times have a finite mixture of TGCD's. The likelihood function based on Type-II censored data, see Lawless [12], is given by

$$
\mathrm{L}(\theta \mid t)=\frac{\mathrm{n} !}{(\mathrm{n}-\mathrm{r}) !}\left[\prod_{\mathrm{i}=1}^{\mathrm{r}} \mathrm{f}_{\Theta}\left(\mathrm{t}_{\mathrm{i}}\right)\left[\mathrm{S}_{\Theta}\left(\mathrm{t}_{\mathrm{r}}\right)\right]^{\mathrm{n}-\mathrm{r}} .\right.
$$

Using the prior (2.8) and the LF (3.6), the posterior PDF of the parameters $\left(p, \alpha_{1}, \alpha_{2}, \gamma_{1}, \gamma_{2}, \beta\right)$ can be written as

$$
\begin{aligned}
& \delta^{*}(\theta \mid t)=\mathrm{p}^{\mathrm{c}_{1}-1}(1-\mathrm{P})^{\mathrm{c}_{2}-1} \alpha_{1}^{\mathrm{c}_{3}+\mathrm{c}_{4}-1} \alpha_{2}^{\mathrm{c}_{6}+\mathrm{c}_{7}-1} \tilde{\mathrm{a}}_{1}^{\mathrm{c}_{3}-1} \tilde{\mathrm{a}}_{2}^{\mathrm{c}_{6}-1} \beta^{\mathrm{c}_{9}-1} \\
& \exp (1-\mathrm{p})^{\mathrm{c}_{2}-1} \alpha_{1}^{\mathrm{c}_{3}+\mathrm{c}_{4}-1} \alpha_{2}^{\mathrm{c}_{6}+\mathrm{c}_{7}-1} \gamma_{1}^{\mathrm{c}_{3}-1} \gamma_{2}^{\mathrm{c}_{6}-1} \beta^{\mathrm{c}_{9}-1} \\
& \exp \left\{-\left[\alpha_{1}\left(\gamma_{1}+\mathrm{c}_{5}\right)+\alpha_{2}\left(\gamma_{2}+\mathrm{c}_{8}\right)+\mathrm{c}_{10} \beta\right]\right\}\left[\Pi_{\mathrm{i}=1}^{\mathrm{r}} \mathrm{f}_{\Theta}\left(\mathrm{t}_{\mathrm{i}}\right)\left[\mathrm{S}_{\Theta}\left(\mathrm{t}_{\mathrm{r}}\right)\right]^{\mathrm{n}-\mathrm{r}} .\right.
\end{aligned}
$$

and the same is done as 3.1 .

\section{Bayes estimation based on progressively type-II censoring scheme}

The progressive type-II censored model is of importance in the field of reliability and life testing. Suppose nidentical units are placed on a lifetime test. At the time of the $i^{\text {th }}$ failure, $R_{i}$ surviving units are randomly withdrawn from the experiment, $1 \leq i \leq r$. Thus, if $r$ failures are observed then $R_{1}+R_{2}+\ldots+R_{r}$, units are progressively censored, hence $n=r+R_{1}+R_{2}+\ldots+R_{r}$ and $T_{1: r: n}^{M}<T_{2: r: n}^{M}<\ldots<T_{r: r: n}^{M}$ describe the progressively censored failure times, where $M=\left(R_{1}, R_{2}, \ldots, R_{r}\right)$ and $\sum_{\mathrm{i}=1}^{\mathrm{r}} \mathrm{R}_{\mathrm{i}}=\mathrm{n}-\mathrm{r}$. The likelihood function based on progressively type-II censored data $t=\left(t_{1: r: n}^{M}, t_{(2: r: n)}^{M}, \ldots, t_{(r: r: n)}^{M}\right)$ which can be written for simplicity as $t=\left(t_{1}, t_{2}, \ldots, t_{r}\right)$ is given by

$$
\mathrm{L}(\theta \mid t)=\mathrm{c} \prod_{i=1}^{r} \mathrm{f}_{\grave{\mathrm{E}}}\left(\mathrm{t}_{\mathrm{i}}\right)\left(\mathrm{S}_{\Theta}\left(\mathrm{t}_{\mathrm{i}}\right)\right)^{R_{\mathrm{i}}},
$$

Where,

$\mathrm{c}=\mathrm{n}\left(\mathrm{n}-\mathrm{R}_{1}-1\right)\left(\mathrm{n}-\mathrm{R}_{1}-\mathrm{R}_{2}-2\right) \ldots\left(\mathrm{n}-\mathrm{R}_{1}-\mathrm{R}_{2}-\ldots-\mathrm{R}_{\mathrm{r}-1}-\mathrm{r}+1\right), \quad$ See Balakrishnan \& Aggarwala [13].

Using the LF (3.8) and the prior PDF (2.8), the posterior PDF will be given by

$$
\begin{aligned}
& \pi^{*}(\theta \mid t)=\mathrm{C}^{*} \mathrm{p}^{\mathrm{c}_{1}-1}(1-\mathrm{p})^{\mathrm{c}_{2}-1} \alpha_{1}^{\mathrm{c}_{3}+\mathrm{c}_{4}-1} \alpha_{2}^{\mathrm{c}_{6}+\mathrm{c}_{7}-1} \gamma_{1}^{\mathrm{c}_{3}-1} \gamma_{2}^{\mathrm{c}_{6}-1} \beta^{\mathrm{c}_{9}-1} \\
& \exp \left\{-\left[\alpha_{1}\left(\gamma_{1}+\mathrm{c}_{5}\right)+\alpha_{2}\left(\gamma_{2}+\mathrm{c}_{8}\right)+\mathrm{c}_{10} \beta\right]\right\} \prod_{i=1}^{r}\left[\mathrm{f}\left(\mathrm{t}_{\mathrm{i}} ; \theta\right)\right]\left[\mathrm{S}\left(\mathrm{t}_{0} ; \theta\right)\right]^{\mathrm{R}_{\mathrm{i}}}
\end{aligned}
$$


Where,

$C^{*}=n\left(n-R_{1}-1\right)\left(n-R_{1}-R_{2}-1\right) \ldots .\left(n-R_{1}-R_{2}-\ldots-R_{r-1}-r-1\right)$, and also the same is done as 3.1 and 3.2

\section{Simulation study and data analysis}

In this section, all studied parameters and functions will be estimated based on type-I, type-II and progressive type-II censoring samples from generated and real data.

\section{Simulation study}

In this section, a simulation study is carried out to study the behavior of the MSE's. In case of $j=2$, we can write $p_{1}=p$, $p_{2}=1-p$, then the vector of all parameters will be in the form $\theta=\left(\alpha_{1}, \alpha_{2}, \beta, \gamma_{1}, \gamma_{2}, p\right)$.

For different values of $t_{0}$ and $r$, follow the following steps:

Making use of the set of hyper parameters, the vector of the population parameters will be generated.

Making use of the generated vector of parameters $\theta=\left(\alpha_{1}, \alpha_{2}, \beta, \gamma_{1}, \gamma_{2}, p\right)$, samples of different sizes $\mathrm{n}(10,20,30)$ are generated from a mixture of two TGCD as follows:

- Generate $\mathrm{U}_{1}$ and $\mathrm{U}_{2}$ from the uniform distribution $\mathrm{U}(0,1)$.

- If $\mathrm{u}_{1}<p$, generate from $\mathrm{F}_{1}\left(\mathrm{t} ; \alpha_{1}, \beta, \gamma_{1}\right)$ using $\mathrm{u}_{2}$, otherwise generate from $\mathrm{F}_{2}\left(\mathrm{t} ; \alpha_{2}, \beta, \gamma_{2}\right)$ using $\mathrm{u}_{2}$.
For a value $\mathrm{t}_{0}$, we consider all values of the random variable $\mathrm{T}$ which are less than or equal $\operatorname{MSE}(\hat{\theta})=\frac{1}{m} \sum_{\mathrm{j}=1}^{m}\left(\hat{\theta}_{j}-\theta\right)^{2}$, (type-I censored sample).

Based on this sample and for different values of $t_{0}$, we can use the Bayes method to obtain the estimate of the vector of parameters $\theta$. survival and hazard rate functions.

Based on the sample $t_{1}<t_{2}<\ldots<t_{r}$ which is a typeII censored sample, we can use Bayes method, as done in case of type-I censoring, to obtain the BE's of the same vector of parameters and functions, for different values of $r$.

For different schemes, progressive type-II will be generated and the same is done based on the generated progressively typeII censored samples.

Repeat steps 1-6 (m) times for different samples.

The MSE's of $\hat{\theta}$ over the m samples is given by:

$$
\operatorname{MSE}(\hat{\theta})=\frac{1}{m} \sum_{\mathbf{j}=1}^{m}\left(\hat{\theta}_{j}-\theta\right)^{2},
$$

Where $\theta$ is the actual value of the vector of parameters $\hat{\theta}_{j}$ and $\theta$ is the estimate of the vector of parameters over the sample $j$.

In Tables 1-6 the BE's of all parameters and functions have been obtained based on Type-I, Type-II and progressively TypeII censored samples.

Table 1: MSE's of the BE's of $\alpha_{1}, \alpha_{2}, \beta, \gamma_{1}, \gamma_{2}$ and $\mathrm{p}$ based on type-I censored data of different sizes $\mathrm{n}$, censoring values $t_{0}$ $\left(\alpha_{1}=2.5, \alpha_{2}=1.6, \beta=1.5, \gamma_{1}=3.5, \gamma_{2}=1.7, p=0.3\right)$.

\begin{tabular}{|c|c|c|c|c|c|c|c|}
\hline$n$ & $t_{0}$ & $\operatorname{MSE}(\hat{p})$ & $\operatorname{MSE}\left(\hat{\alpha_{1}}\right)$ & $\operatorname{MSE}\left(\hat{\alpha_{2}}\right)$ & $\operatorname{MSE}(\hat{\beta})$ & $\operatorname{MSE}\left(\hat{\gamma_{1}}\right)$ & $\operatorname{MSE}\left(\hat{\gamma_{2}}\right)$ \\
\hline \multirow{3}{*}{10} & 2.5 & 0.0027 & 0.0652 & 0.0371 & 0.0291 & 0.04416 & 0.0819 \\
\cline { 2 - 8 } & 4.5 & 0.0024 & 0.0518 & 0.0351 & 0.0281 & 0.04194 & 0.0751 \\
\cline { 2 - 8 } & 6 & 0.0021 & 0.0501 & 0.0331 & 0.0221 & 0.03719 & 0.0681 \\
\hline \multirow{3}{*}{20} & 2.5 & 0.0023 & 0.0441 & 0.0214 & 0.0104 & 0.03098 & 0.0916 \\
\cline { 2 - 8 } & 4.5 & 0.0019 & 0.0221 & 0.0201 & 0.0061 & 0.02997 & 0.0414 \\
\hline \multirow{3}{*}{30} & 6 & 0.0015 & 0.0179 & 0.0193 & 0.0042 & 0.02154 & 0.0251 \\
\cline { 2 - 8 } & 2.5 & 0.0014 & 0.0192 & 0.0177 & 0.0037 & 0.02196 & 0.0917 \\
\cline { 2 - 8 } & 4.5 & 0.0009 & 0.0104 & 0.0127 & 0.0021 & 0.02015 & 0.0421 \\
\hline
\end{tabular}

Table 2: MSE's of the BE's of $S\left(t^{*}\right)$ and $h\left(t^{*}\right)$ based on type-I censored data of different sizes $\mathrm{n}$, censoring values $t_{0}$ $\left(\alpha_{1}=2.5, \alpha_{2}=1.6, \beta=1.5, \gamma_{1}=3.5, \gamma_{2}=1.7, p=0.3\right) . S\left(t^{*}\right)=0.259437, h\left(t^{*}\right)=0.964188, t^{*}=3.0$.

\begin{tabular}{|c|c|c|c|c|c|}
\hline$n$ & $t_{0}$ & $\overline{\overline{S\left(t^{*}\right)}}$ & $\overline{\widehat{h\left(t^{*}\right)}}$ & $\operatorname{MSE} \overline{\overline{\left(S\left(t^{*}\right)\right)}}$ & $\operatorname{MSE} \overline{\overline{\left(H\left(t^{*}\right)\right)}}$ \\
\hline \multirow{3}{*}{10} & 2.5 & 0.21098 & 0.9612 & 0.002108 & 0.001412 \\
\hline & 4.5 & 0.21008 & 0.9511 & 0.002011 & 0.001109 \\
\hline & 6 & 0.22098 & 0.959 & 0.001901 & 0.001031 \\
\hline \multirow{3}{*}{20} & 2.5 & 0.22156 & 0.9501 & 0.002013 & 0.000916 \\
\hline & 4.5 & 0.23128 & 0.9591 & 0.001987 & 0.000519 \\
\hline & 6 & 0.23891 & 0.961 & 0.001517 & 0.000318 \\
\hline
\end{tabular}




\section{Biostatistics and Biometrics Open Access Journal}

\begin{tabular}{|c|c|c|c|c|c|}
\hline \multirow{3}{*}{30} & 2.5 & 0.25125 & 0.959 & 0.001929 & 0.000615 \\
\cline { 2 - 7 } & 4.5 & 0.25192 & 0.9621 & 0.001615 & 0.000581 \\
\cline { 2 - 7 } & 6 & 0.26182 & 0.9615 & 0.001016 & 0.000505 \\
\hline
\end{tabular}

Table 3: MSE's of the BE's of $\alpha_{1}, \alpha_{2}, \beta, \gamma_{1}, \gamma_{2}$ and $\mathrm{p}$ based on type-ll censored data of different sizes $\mathrm{n}$, censoring values $\left(\alpha_{1}=2.5, \alpha_{2}=1.6, \beta=1.5, \gamma_{1}=3.5, \gamma_{2}=1.7, p=0.3\right)$.

\begin{tabular}{|c|c|c|c|c|c|c|c|}
\hline$n$ & $r$ & $\operatorname{MSE}(\hat{p})$ & $\operatorname{MSE}\left(\hat{\alpha}_{1}\right)$ & $\operatorname{MSE}\left(\hat{\alpha_{2}}\right)$ & $\operatorname{MSE}(\hat{\beta})$ & $\operatorname{MSE}\left(\hat{\gamma_{1}}\right)$ & $\operatorname{MSE}\left(\hat{\gamma_{2}}\right)$ \\
\hline 10 & 5 & 0.0031 & 0.0418 & 0.0331 & 0.0217 & 0.0424 & 0.1081 \\
\hline & 10 & 0.0027 & 0.0397 & 0.0294 & 0.0211 & 0.0373 & 0.0715 \\
\hline 20 & 5 & 0.0028 & 0.0407 & 0.0221 & 0.0061 & 0.0291 & 0.0712 \\
\hline 30 & 10 & 0.0021 & 0.0383 & 0.0219 & 0.0032 & 0.0261 & 0.0671 \\
\hline & 15 & 0.0018 & 0.0311 & 0.0208 & 0.0028 & 0.0208 & 0.0409 \\
\hline & 20 & 0.0022 & 0.0209 & 0.0142 & 0.0018 & 0.0221 & 0.0441 \\
\hline & 30 & 0.0008 & 0.0143 & 0.0103 & 0.0009 & 0.0152 & 0.0106 \\
\hline
\end{tabular}

Table 4: MSE's of the BE's of $S(t *)$ and $h(t *)$ based on type-II censored data of different sizes $\mathrm{n}$, censoring values $r$ $\left(\alpha_{1}=2.5, \alpha_{2}=1.6, \beta=1.5, \gamma_{1}=3.5, \gamma_{2}=1.7, p=0.3\right) . S\left(t^{*}\right)=0.259437, h(t *)=0.964188, t^{*}=3.0$. .

\begin{tabular}{|c|c|c|c|c|c|}
\hline$n$ & $r$ & $\overline{\overline{S\left(t^{*}\right)}}$ & $\overline{\overline{h\left(t^{*}\right)}}$ & $\operatorname{MSE} \overline{\overline{\left(S\left(t^{*}\right)\right)}}$ & $\operatorname{MSE} \overline{\overline{\left(h\left(t^{*}\right)\right)}}$ \\
\hline \multirow{2}{*}{10} & 5 & 0.2812 & 0.9501 & 0.0025 & 0.0108 \\
\hline & 10 & 0.2781 & 0.9521 & 0.0022 & 0.0101 \\
\hline \multirow{3}{*}{20} & 5 & 0.2412 & 0.9491 & 0.0016 & 0.0008 \\
\hline & 10 & 0.2421 & 0.9503 & 0.0009 & 0.0004 \\
\hline & 15 & 0.2398 & 0.958 & 0.0005 & 0.0001 \\
\hline \multirow{3}{*}{30} & 15 & 0.2536 & 0.9501 & 0.0002 & 0.00009 \\
\hline & 20 & 0.2543 & 0.9605 & 0.0001 & 0.00004 \\
\hline & 30 & 0.2589 & 0.9601 & 0.00006 & 0.00002 \\
\hline
\end{tabular}

Table 5: MSE's of the BE's of $\alpha_{1}, \alpha_{2}, \beta, \gamma_{1}, \gamma_{2}$ and $\mathrm{p}$ based on progressively type-Il censored data of different sizes $\mathrm{n}$, censoring schemes $\mathrm{M}$.

$M_{1}=(0,5,3,2,2,1,0,0,2,0)$

$M_{2}=(0,3,1,0,1,0,0,2,1,0,0,1,0,1,0)$

$M_{3}=(0,0,0,0,0,0,0,0,0,0,0,0,0,0,0,0,0,0,0,0,0,0,0,0,0)$.

$\left(\alpha_{1}=2.5, \alpha_{2}=1.6, \beta=1.5, \gamma_{1}=3.5, \gamma_{2}=1.7, p=0.3\right)$.

\begin{tabular}{|c|c|c|c|c|c|c|c|}
\hline$n$ & $(r, M)$ & $\operatorname{MSE}(\hat{p})$ & $\operatorname{MSE}\left(\hat{\alpha}_{1}\right)$ & $\operatorname{MSE}\left(\hat{\alpha}_{2}\right)$ & $\operatorname{MSE}(\hat{\beta})$ & $\operatorname{MSE}\left(\hat{\gamma_{1}}\right)$ & $\operatorname{MSE}\left(\hat{\gamma_{2}}\right)$ \\
\hline \multirow{3}{*}{25} & $\left(10, M_{1}\right)$ & 0.0027 & 0.0501 & 0.0351 & 0.0207 & 0.05162 & 0.08142 \\
\cline { 2 - 7 } & $\left(15, M_{2}\right)$ & 0.0019 & 0.0481 & 0.0321 & 0.0183 & 0.04417 & 0.07916 \\
\cline { 2 - 7 } & $\left(25, M_{3}\right)$ & 0.0011 & 0.0448 & 0.0305 & 0.0034 & 0.03012 \\
\hline
\end{tabular}


Table 6: MSE's of the BE's of $S\left(t^{*}\right)$ and $h\left(t^{*}\right)$ based on progressively type-Il censored data of different sizes $\mathrm{n}$, censoring schemes M. $M_{1}=(0,5,3,2,2,1,0,0,2,0)$

$M_{2}=(0,3,1,0,1,0,0,2,1,0,0,1,0,1,0)$

$M_{3}=(0,0,0,0,0,0,0,0,0,0,0,0,0,0,0,0,0,0,0,0,0,0,0,0,0)$.

$\left(\alpha_{1}=2.5, \alpha_{2}=1.6, \beta=1.5, \gamma_{1}=3.5, \gamma_{2}=1.7, p=0.3\right)$.

$S\left(t^{*}\right)=0.259437, h\left(t^{*}\right)=0.964188, t^{*}=3.0$.

\begin{tabular}{|c|c|c|c|c|c|}
\hline$n$ & $(r, M)$ & $\overline{\overline{S\left(t^{*}\right)}}$ & $\overline{\overline{h\left(t^{*}\right)}}$ & $\overline{M S E} \overline{\overline{\left(S\left(t^{*}\right)\right)}}$ & $M S E \overline{\overline{\left(h\left(t^{*}\right)\right)}}$ \\
\hline \multirow{3}{*}{25} & $\left(10, M_{1}\right)$ & 0.2471 & 0.9512 & 0.0042 & 0.0027 \\
\cline { 2 - 6 } & $\left(15, M_{2}\right)$ & 0.2506 & 0.9445 & 0.0038 & 0.0021 \\
\cline { 2 - 6 } & $\left(25, M_{3}\right)$ & 0.2522 & 0.9517 & 0.0028 & 0.0018 \\
\hline
\end{tabular}

Data analysis

In this section, a mixture of two real data sets from Ateya \& Madhagi [14] is introduced. These data are (after ordering)

$2.3707,2.4282,2.4743,2.4858,2.4858,2.5088,2.5663$, $2.6239,2.6239,2.6814,2.762,3.0266,3.1187,3.1509,3.5905$, $3.6825,3.6825,3.7136,3.7373,3.7799,3.8322,3.8603$, 3.8981, 3.9644, 4.059, 4.1583, 4.2577, 4.2719, 4.3326, 4.3846, $4.4275,4.4275,4.532,4.554,4.5856,4.6172,4.6172,4.6488$, 4.7121, 4.7355, 4.807, 5.1518, 5.166, 5.1944, 5.2369, 5.6201, 5.9607, 6.5568, 7.1529, 7.644, 7.81, 7.84, 7.938, 8.0044, 8.134, $8.526,8.82,8.82,9.31,9.31,9.506,9.8,10,10.001,10.1,10.3$, $10.9504,11.3302,11.3935,11.6,11.8394,12.6457,12.9286$, $13.1,13.169,13.2,13.3246,13.4,13.7,13.7914,13.8,14,14,14$, $14.0177,14.0885,14.1,14.1733,14.4,14.6,14.6118,15.0645$, $15.5,15.5454,15.9698,16.1,16.479,16.5,16.9,16.9,17.0306$,
$17.1,17.2,17.3,17.3,17.3,17.3,17.4,17.7,17.8,17.8793$, $17.9,18.2,18.9,19.2357,19.4,20.0812,21.5,22.7587,23.4$, 23.6, 24.1, 25, 26.4226, 26.5, 26.7, 26.7, 26.8, 26.9, 27.6, 28.4, 28.9, 29.4, 29.8, 30, 30.157, 30.4, 30.9, 31.2, 31.8, 32.9, 33.9, 34.4551, 35.5, 37.6963, 37.7, 39.8, 40.5852, 41.1, 42.5, 44.6719, 46.2, 48.829, 51.9, 54.3249, 55.5, 58.2, 60.3141, 62.1, 65.5281, 67.2192, 67.7, 72.4151, 76.7, 79.8316, 85.5389, 86.3, 92.0212, $94,97.6,99.2082,101.3,105.3,106,107.6,107.9,109.3,110$, $110.5,112.8,115.4,118.5,120,120.3,120.5,121.9,124.1,126.1$, $131.3,133.8,135.4,137.9,139.5,141.9,142.8,145.8,146.5$, $149.7,150.6,153.5,158.6,161.3,163.9,168.3,174,176.7,180.9$, $184.3,190.3,196.8$

The BE's of the parameters, survival and hazard rate functions based on type-I, type-II and progressively type-II under the previous real data set are summarized in Tables 7-12.

Table 7: BE's of the population parameters based on type-I censoring scheme from real data set.

\begin{tabular}{|c|c|c|c|c|c|c|c|}
\hline$n$ & $t_{0}$ & $\hat{p}$ & $\hat{\alpha}_{1}$ & $\hat{\alpha}_{2}$ & $\hat{\beta}$ & $\hat{\gamma}_{1}$ & $\hat{\gamma}_{2}$ \\
\hline \multirow{3}{*}{210} & 30 & 0.28162 & 4.97078 & 10.2716 & 2.3231 & 3713.72 & 4132.99 \\
\cline { 2 - 8 } & 90 & 0.28618 & 5.16162 & 10.3415 & 2.3651 & 3733.21 & 4267.65 \\
\cline { 2 - 8 } & 200 & 0.29017 & 5.12739 & 10.3651 & 2.4015 & 3731.87 & 4254.87 \\
\hline
\end{tabular}

Table 8: BE's of the survival and hazard rate functions based on type-I censoring scheme from real data set at

\begin{tabular}{|c|c|c|c|}
\hline$n$ & $t_{0}$ & $\overline{\widehat{S\left(t^{*}\right)}}$ & $\overline{\overline{h\left(t^{*}\right)}}$ \\
\hline \multirow{2}{*}{210} & 30 & 0.937385 & 0.000858 \\
\cline { 2 - 4 } & 90 & 0.940918 & 0.000887 \\
\cline { 2 - 4 } & 200 & 0.939715 & 0.000898 \\
\hline
\end{tabular}

Table 9: BE's of the population parameters based on type-II censoring scheme from real data set.

\begin{tabular}{|c|c|c|c|c|c|c|c|}
\hline$n$ & $\boldsymbol{r}$ & $\hat{p}$ & $\hat{\alpha}_{1}$ & $\hat{\alpha}_{2}$ & $\hat{\beta}$ & $\hat{\gamma}_{1}$ & $\hat{\gamma}_{2}$ \\
\hline \multirow{3}{*}{210} & 70 & 0.271260 & 4.93372 & 10.55165 & 2.4098 & 3689.32 & 4217.09 \\
\cline { 2 - 9 } & 140 & 0.27701 & 5.32415 & 10.41524 & 2.3908 & 3701.76 & 4231.82 \\
\cline { 2 - 9 } & 210 & 0.28971 & 5.11902 & 10.29081 & 2.3991 & 3781.18 & 4271.93 \\
\hline
\end{tabular}




\section{Biostatistics and Biometrics Open Access Journal}

Table 10: BE's of the survival and hazard rate functions based on type-II censoring scheme from real data set at

\begin{tabular}{|c|c|c|c|}
\hline$n$ & $\hat{\gamma}_{2}$ & $\overline{\overline{S\left(t^{*}\right)}}$ & $\overline{\overline{h\left(t^{*}\right)}}$ \\
\hline 210 & 70 & 0.929412 & 0.000839082 \\
\hline & 140 & 0.938919 & 0.000861827 \\
\hline
\end{tabular}

Table 11: BE's of the population parameters based on progressively type-ll censoring scheme from real data set.

\begin{tabular}{|c|c|c|c|c|c|c|c|}
\hline$n$ & $(r, M)$ & $\hat{p}$ & $\hat{\alpha}_{1}$ & $\hat{\alpha}_{2}$ & $\hat{\beta}$ & $\hat{\gamma}_{1}$ & $\hat{\gamma}_{2}$ \\
\hline \multirow{3}{*}{210} & $(75, \mathrm{M} 1)$ & 0.26901 & 4.87159 & 10.71287 & 2.3291 & 3644.95 & 4180.15 \\
\cline { 2 - 9 } & $(150, \mathrm{M} 2)$ & 0.27109 & 5.21782 & 10.51527 & 2.3781 & 3741.98 & 4261.54 \\
\cline { 2 - 9 } & $(210, \mathrm{M} 3)$ & 0.28017 & 5.10082 & 10.33162 & 2.4055 & 3750.15 & 4197.92 \\
\hline
\end{tabular}

$M 1=(12,017,30,020,27,015,22,07,35,06,9,04)$

$M 2=(10,030,20,020,20,030,5,020,2,030,3,014)$

$M 3=(0210)$

Table 12: BE's of the survival and hazard rate functions based on progressively type-II censoring scheme from real data set.

\begin{tabular}{|c|c|c|c|}
\hline$n$ & $(r, M)$ & $\overline{\overline{S\left(t^{*}\right)}}$ & $\overline{\overline{h\left(t^{*}\right)}}$ \\
\hline \multirow{2}{*}{210} & $(75, \mathrm{M} 1)$ & 0.918273 & 0.000821625 \\
\cline { 2 - 4 } & $(150, \mathrm{M} 2)$ & 0.925612 & 0.000832154 \\
\cline { 2 - 4 } & $(210, \mathrm{M} 3)$ & 0.932012 & 0.000884428 \\
\hline
\end{tabular}

data set.

$M 1=(12,017,30,020,27,015,22,07,35,06,9,04)$

$M 2=(10,030,20,020,20,030,5,020,2,030,3,014)$

$M 3=(0210)$

In most cases, observe the following:

- $\quad$ For fixed and and by increasing the sample size n, we often get smaller MSE' s.

- $\quad$ For fixed sample size $\mathrm{n}$ and by increasing the censoring values fixed t_0and $r$, we often get smaller MSE' s.

- The largest values of and in each case represent the complete sample case.

- In progressively type II, for fixed $\mathrm{n}$, we often get smaller MSE' s by increasing $r$.

\section{References}

1. Johnson NL, Kotz S, Balakrishnan N (1995) Continuous Univariate Distributions, V-2, ( $2^{\text {nd }}$ edn) edition, Wiley, New York, USA Pg No. 784.

2. Ateya SF, AL-Hussaini, EK (2012) On truncated generalized Cauchy distribution. J Math Comput Sci 2: 289-304.

3. Ahsanullah M (2015) Some Inferences on Truncated Generalized Cauchy Distribution. AfrikaStatistika 10: 815-826.

4. McLachlan GJ, Peel D( 2000) Finite Mixture Models. Wiley, New York, USA.

5. Titterington DM, Smith AFM, Makov UE (1985) Statistical Analysis of Finite Mixture Distributions. Wiley, New York, USA
6. Bozidar VP, Gauss C, Edwin MO, Marcelino ARP (2017) A new extended mixture normal distribution. Math Commun 22: 53-73.

7. Satheesh KC, Manju L (2017) A Note on Logistic Mixture Distributions Biostat Biometrics Open Acc J 2(5): BBOAJ.MS.ID.555598.

8. Jaheen ZF, Al-Harbi, MM (2011) Bayesian estimation for the exponentiated Weibull model via Markov chain Monte Carlo simulation. Communications in Statistics: Simulation and Computation 40: 532543.

9. Press SJ (2003) Subjective and Objective Bayesian Statistics: Principles, Models and Applications, Wiley, New York.

10. Upadhyaya SK, Vasishta N, Smith AFM (2001) Bayes inference in life testing and reliability via Markov chain Monte Carlo simulation. Sankhya A 63: 15-40.

11. Upadhyaya SK, Gupta A (2010) A Bayes analysis of modified Weibull distribution via Markov chain Monte Carlo simulation. J of Statist Comput and Simul 80: 241-254

12. Lawless JF (2003) Statistical Models and Methods for Lifetime Data $\left(2^{\text {nd }}\right.$ edn) Wiley, New York, USA.

13. Balakrishnan N, Aggarwala R (2000) Progressive Censoring: Theory, Methods, and Applications. Springer.

14. Ateya SF, Elham A Madhagi (2013) On multivariate truncated generalized Cauchy distribution. Statistical Papers 54: 879-897. 
This work is licensed under Creative Commons Attribution 4.0 Licens

DOI: 10.19080/BBOAJ.2018.05.555652

\section{Your next submission with Juniper Publishers} will reach you the below assets

- Quality Editorial service

- Swift Peer Review

- Reprints availability

- E-prints Service

- Manuscript Podcast for convenient understanding

- Global attainment for your research

- Manuscript accessibility in different formats

( Pdf, E-pub, Full Text, Audio)

- Unceasing customer service

Track the below URL for one-step submission https://juniperpublishers.com/online-submission.php 